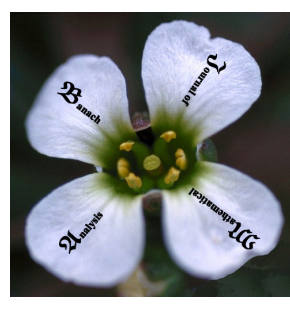

Banach J. Math. Anal. 2 (2008), no. 2, 9-15

B anach J ournal of $\mathbf{M}_{\text {athematical }} \mathbf{A}_{\text {nalysis }}$

ISSN: $1735-8787$ (electronic)

http://www.math-analysis.org

\title{
HARDY INEQUALITY OF FRACTIONAL ORDER
}

\author{
PETR GURKA ${ }^{1}$ AND BOHUMÍR OPIC ${ }^{2 *}$ \\ This paper is dedicated to Professor Josip E. Pečarić
}

Submitted by M. Abel

ABStRACT. We prove optimality of power-type weights in the Hardy inequality of fractional order.

\section{InTRODUCTION AND THE MAIN RESULT}

In [3] the following theorem was proved.

Theorem 1.1. Let $1 \leq p<\infty, \delta \in(0,1) \cup(1, p)$ and $u$ be a locally integrable function on $[0, \infty)$. Let

(i) either $0<\delta<1$ and $\lim _{t \rightarrow \infty} \frac{1}{t} \int_{0}^{t} u=0$,

(ii) or $1<\delta<p$ and $\lim _{t \rightarrow 0_{+}} \frac{1}{t} \int_{0}^{t} u=0$.

Then

$$
\int_{0}^{\infty}|u(x)|^{p} x^{-\delta} \mathrm{d} x \leq C \int_{0}^{\infty} \int_{0}^{\infty} \frac{|u(x)-u(y)|^{p}}{|x-y|^{\delta+1}} \mathrm{~d} x \mathrm{~d} y,
$$

where $C=(1+p /|\delta-1|)^{p} / 2$.

Date: Received: 29 February 2008; Accepted: 25 March 2008.

* Corresponding author.

2000 Mathematics Subject Classification. 26D10.

Key words and phrases. Hardy inequality of fractional order, power-type weights.

The research was partly supported by grant no. 201/05/2033 of the Grant Agency of the Czech Republic and by the Institutional Research Plan no. AV0Z10190503 of the Academy of Sciences of the Czech Republic. 
It is known that the restriction $\delta \in(0,1) \cup(1, p)$ is essential. Indeed, if either $\delta \leq 0$ or $\delta \geq p$, then the integral on the right-hand side of (1.1) diverges for each nonzero function $u \in C_{0}^{\infty}(0, \infty)$. If $p>1$ and $\delta=1$, then there is no finite constant $C$ such that inequality (1.1) holds for all functions in question. Indeed, inserting the functions

$$
u_{\varepsilon}(t)=\frac{t-\varepsilon}{\varepsilon} \chi_{[\varepsilon, 2 \varepsilon)}(t)+\chi_{[2 \varepsilon, 1 / 2)}(t)+2(1-t) \chi_{(1 / 2,1)}(t)
$$

into (1.1) and letting $\varepsilon \rightarrow 0_{+}$, we obtain that the constant $C \rightarrow \infty$. (See [3, Remark 6].) Here the symbol $\chi_{I}$ stands for the characteristic function of an interval $I \subset \mathbb{R}$.

The aim of this paper is to show that power-type weights in inequality (1.1) are optimally chosen. This follows from the next result.

Theorem 1.2. Let $1 \leq p<\infty$. Suppose that $\delta \in(0,1) \cup(1, p), \eta \in(0, p)$ and there is a positive constant $C$ such that the inequality

$$
\int_{0}^{\infty}|u(x)|^{p} x^{-\delta} \mathrm{d} x \leq C \int_{0}^{\infty} \int_{0}^{\infty} \frac{|u(x)-u(y)|^{p}}{|x-y|^{\eta+1}} \mathrm{~d} x \mathrm{~d} y
$$

holds for all locally integrable functions u satisfying one of conditions (ii), (ii) of Theorem 1.1. Then $\eta=\delta$.

The proof of Theorem 1.2 is based on some ideas developed in [1] and [2].

\section{Proof of Theorem 1.2}

To prove Theorem 1.2 we need several lemmas.

Lemma 2.1. Let $0<p<\infty$ and $w$ be a measurable nonnegative even function. Then

$$
\begin{aligned}
\int_{0}^{\infty} \int_{0}^{\infty}|g(x)-g(y)|^{p} w & (x-y) \mathrm{d} x \mathrm{~d} y \\
= & 2 \int_{0}^{\infty}\left(\int_{0}^{\infty}|g(y+h)-g(y)|^{p} \mathrm{~d} y\right) w(h) \mathrm{d} h
\end{aligned}
$$

provided that the left-hand side of the equality makes sense.

Proof. Using the change of variables $x=y+h$ in the inner integral and applying the Fubini theorem, we obtain

$$
\begin{gathered}
\int_{0}^{\infty} \int_{0}^{\infty}|g(x)-g(y)|^{p} w(x-y) \mathrm{d} x \mathrm{~d} y=\int_{0}^{\infty}\left(\int_{-y}^{\infty}|g(y+h)-g(y)|^{p} w(h) \mathrm{d} h\right) \mathrm{d} y \\
=\int_{0}^{\infty}\left(\int_{0}^{\infty}|g(y+h)-g(y)|^{p} \mathrm{~d} y\right) w(h) \mathrm{d} h \\
\quad+\int_{-\infty}^{0}\left(\int_{-h}^{\infty}|g(y+h)-g(y)|^{p} \mathrm{~d} y\right) w(h) \mathrm{d} h
\end{gathered}
$$


In the second term we replace $h$ by $k$ and $y$ by $z$, then we make two changes of variables $h=-k$ and $z-h=y$ and use the fact that $w(-h)=w(h)$, to arrive at

$$
\int_{-\infty}^{0}\left(\int_{-h}^{\infty}|g(y+h)-g(y)|^{p} \mathrm{~d} y\right) w(h) \mathrm{d} h=\int_{0}^{\infty}\left(\int_{0}^{\infty}|g(y+h)-g(y)|^{p} \mathrm{~d} y\right) w(h) \mathrm{d} h .
$$

Together with (2.2), it gives 2.1).

In what follows we write $A \lesssim B$ (or $A \gtrsim B$ ) if $A \leq c B$ (or $c A \geq B$ ) for some positive constant $c$ independent of appropriate quantities involved in the expressions $A$ and $B$. For $p \in[1, \infty]$, the conjugate number $p^{\prime}$ is defined by $1 / p+1 / p^{\prime}=1$ with the convention that $1 / \infty=0$.

Lemma 2.2. Let $w$ be a measurable nonnegative function, let $p \in[1, \infty), \alpha \in$ $(1, \infty)$ and $\alpha^{\prime}:=\alpha /(\alpha-1)$. Then

$$
\begin{aligned}
\int_{0}^{\infty} & \left(\int_{0}^{\infty}|g(y+h)-g(y)|^{p} \mathrm{~d} y\right) w(h) \mathrm{d} h \\
& \lesssim \int_{0}^{\infty}\left(\int_{0}^{2 h}|g(y)|^{p} \mathrm{~d} y\right) w(h) \mathrm{d} h+\int_{0}^{\infty}\left(\int_{h}^{\infty}\left|g^{\prime}(y)\right|^{p} \mathrm{~d} y\right) h^{p} w(h) \mathrm{d} h
\end{aligned}
$$

and

$$
\begin{aligned}
\int_{0}^{\infty}\left(\int_{0}^{\infty}|g(y+h)-g(y)|^{p} \mathrm{~d} y\right) w(h) \mathrm{d} h & \\
\lesssim & \int_{0}^{\infty}\left(\int_{0}^{h}\left(\int_{y}^{\infty}\left|g^{\prime}(\tau)\right|^{\alpha} \mathrm{d} \tau\right)^{p / \alpha} \mathrm{d} y\right) h^{p / \alpha^{\prime}} w(h) \mathrm{d} h \\
& \quad+\int_{0}^{\infty}\left(\int_{h}^{\infty}\left|g^{\prime}(y)\right|^{p} \mathrm{~d} y\right) h^{p} w(h) \mathrm{d} h
\end{aligned}
$$

for all locally absolutely continuous functions $g$ on $[0, \infty)$.

Proof. Let $h>0$. Then

$$
\begin{aligned}
& \int_{0}^{\infty}|g(y+h)-g(y)|^{p} \mathrm{~d} y \\
&=\int_{0}^{h}|g(y+h)-g(y)|^{p} \mathrm{~d} y+\int_{h}^{\infty}|g(y+h)-g(y)|^{p} \mathrm{~d} y \\
&=: N_{1}(h)+N_{2}(h) .
\end{aligned}
$$

First, we estimate $N_{1}$ :

$$
\begin{aligned}
N_{1}(h)=\int_{0}^{h}|g(y+h)-g(y)|^{p} \mathrm{~d} y & \\
& \lesssim \int_{0}^{h}|g(y+h)|^{p} \mathrm{~d} y+\int_{0}^{h}|g(y)|^{p} \mathrm{~d} y=\int_{0}^{2 h}|g(y)|^{p} \mathrm{~d} y .
\end{aligned}
$$


For the alternative estimate, we use the Hölder inequality with the exponents $\alpha$ and $\alpha^{\prime}$ to get, for all $y>0$,

$$
\begin{aligned}
|g(y+h)-g(y)| & =\left|\int_{y}^{y+h} g^{\prime}(\tau) \mathrm{d} \tau\right| \\
& \leq h^{1 / \alpha^{\prime}}\left(\int_{y}^{y+h}\left|g^{\prime}(\tau)\right|^{\alpha} \mathrm{d} \tau\right)^{1 / \alpha} \leq h^{1 / \alpha^{\prime}}\left(\int_{y}^{\infty}\left|g^{\prime}(\tau)\right|^{\alpha} \mathrm{d} \tau\right)^{1 / \alpha} .
\end{aligned}
$$

Consequently,

$$
N_{1}(h) \leq h^{p / \alpha^{\prime}} \int_{0}^{h}\left(\int_{y}^{\infty}\left|g^{\prime}(\tau)\right|^{\alpha} \mathrm{d} \tau\right)^{p / \alpha} \mathrm{d} y .
$$

Now, we estimate the second term $N_{2}$. We use the estimate $|g(y+h)-g(y)| \leq$ $h \int_{0}^{1}\left|g^{\prime}(y+\tau h)\right| \mathrm{d} \tau$, then the Hölder inequality, the Fubini theorem and the change of variables $y+\tau h=z$ to obtain

$$
\begin{array}{r}
N_{2}(h)=\int_{h}^{\infty}|g(y+h)-g(y)|^{p} \mathrm{~d} y \leq \int_{h}^{\infty} h^{p}\left(\int_{0}^{1}\left|g^{\prime}(y+\tau h)\right| \mathrm{d} \tau\right)^{p} \mathrm{~d} y \\
\leq h^{p} \int_{h}^{\infty}\left(\int_{0}^{1}\left|g^{\prime}(y+\tau h)\right|^{p} \mathrm{~d} \tau\right) \mathrm{d} y=h^{p} \int_{0}^{1}\left(\int_{h(1+\tau)}^{\infty}\left|g^{\prime}(z)\right|^{p} \mathrm{~d} z\right) \mathrm{d} \tau \\
\leq h^{p} \int_{0}^{1}\left(\int_{h}^{\infty}\left|g^{\prime}(z)\right|^{p} \mathrm{~d} z\right) \mathrm{d} \tau=h^{p} \int_{h}^{\infty}\left|g^{\prime}(y)\right|^{p} \mathrm{~d} y .
\end{array}
$$

Estimate (2.3) follows from (2.5), 2.6) and (2.8), estimate (2.4) is a consequence of 2.5, 2.7 and 2.8.

Take $R \in(0, \infty)$ and put

$$
u_{R}(x):=\varphi_{R}(x) \int_{0}^{x} \chi_{(R, 2 R)}(t) t^{-2} \mathrm{~d} t, \quad x \in(0, \infty),
$$

where $\varphi_{R} \in C^{\infty}[0, \infty)$ is a cut-off function such that

$$
\begin{gathered}
\operatorname{supp} \varphi_{R} \subset[0,4 R], \quad 0 \leq \varphi_{R} \leq 1, \\
\varphi_{R}(x)=1 \text { for } x \in[0,3 R], \quad \varphi_{R}(x)=0 \text { for } x \in[4 R, \infty], \\
\left|\varphi_{R}^{\prime}\right| \lesssim R^{-1} \chi_{[3 R, 4 R]} .
\end{gathered}
$$

Obviously,

$$
\begin{array}{r}
\lim _{t \rightarrow \infty} \frac{1}{t} \int_{0}^{t} u_{R}(x) \mathrm{d} x=0 \quad \text { and } \quad \lim _{t \rightarrow 0_{+}} \frac{1}{t} \int_{0}^{t} u_{R}(x) \mathrm{d} x=0, \\
\left|u_{R}^{\prime}(x)\right| \lesssim R^{-2} \chi_{[3 R, 4 R]}(x)+\chi_{(R, 2 R)}(x) x^{-2} \text { for all } x \in(0, \infty) .
\end{array}
$$

Lemma 2.3. Let $1 \leq p<\infty$ and $\delta \in(0,1) \cup(1, p)$. Assume that $u_{R}$ is given by (2.9). Then

$$
\int_{0}^{\infty}\left|u_{R}(x)\right|^{p} x^{-\delta} \mathrm{d} x \gtrsim R^{1-p-\delta} \quad \text { for all } R \in(0, \infty)
$$


Proof. Since

$$
u_{R}(x)=\left\{\begin{array}{ccl}
0 & \text { if } & x \in[0, R] \\
1 / R-1 / x & \text { if } & x \in(R, 2 R] \\
1 /(2 R) & \text { if } & x \in(2 R, 3 R)
\end{array}\right.
$$

we obtain

$$
\int_{0}^{\infty}\left|u_{R}(x)\right|^{p} x^{-\delta} \mathrm{d} x \geq \int_{2 R}^{3 R}(1 /(2 R))^{p} x^{-\delta} \mathrm{d} x \approx R^{1-p-\delta}
$$

and 2.12 is verified.

Lemma 2.4. Suppose that $1 \leq p<\infty$ and $\eta \in(0, p)$. Let $u_{R}$ be given by $(2.9)$. Then

$$
\int_{0}^{\infty} \int_{0}^{\infty} \frac{\left|u_{R}(x)-u_{R}(y)\right|^{p}}{|x-y|^{\eta+1}} \mathrm{~d} x \mathrm{~d} y \lesssim R^{1-p-\eta} \quad \text { for all } R \in(0, \infty) .
$$

Proof. We start with some auxiliary estimates. If $\beta \in[1, \infty)$, then, by $(2.11)$,

$$
\int_{h}^{\infty}\left|u_{R}^{\prime}(t)\right|^{\beta} \mathrm{d} t \lesssim\left\{\begin{array}{cll}
R^{1-2 \beta} & \text { if } & h \in[0,4 R] \\
0 & \text { if } & h \in(4 R, \infty)
\end{array}\right.
$$

Using this estimate with $\beta=p$, the facts that $p \in[1, \infty)$ and $\eta \in(0, p)$, we obtain

$$
\begin{array}{r}
\int_{0}^{\infty}\left(\int_{h}^{\infty}\left|u_{R}^{\prime}(t)\right|^{p} \mathrm{~d} t\right) h^{p-\eta-1} \mathrm{~d} h \lesssim \int_{0}^{\infty} R^{1-2 p} \chi_{(0,4 R]}(h) h^{p-\eta-1} \mathrm{~d} h \\
=R^{1-2 p} \int_{0}^{4 R} h^{p-\eta-1} \mathrm{~d} h \approx R^{1-p-\eta} \quad \text { for all } R \in(0, \infty) .
\end{array}
$$

If $\eta \in(1, p)$, we use 2.13 to get

$$
\begin{aligned}
\int_{0}^{\infty}\left(\int_{0}^{2 h}\left|u_{R}(t)\right|^{p} \mathrm{~d} t\right) & h^{-\eta-1} \mathrm{~d} h \lesssim \int_{R / 2}^{\infty}\left(\int_{0}^{2 h} R^{-p} \mathrm{~d} t\right) h^{-\eta-1} \mathrm{~d} h \\
& \approx R^{-p} \int_{R / 2}^{\infty} h^{-\eta} \mathrm{d} h \approx R^{1-p-\eta} \quad \text { for all } R \in(0, \infty)
\end{aligned}
$$

Now, assume that $\eta \in(0,1]$ and $\alpha \in(1, \infty)$ is such that $\alpha^{\prime}>p / \eta$. Then

$$
0>p / \alpha^{\prime}-\eta>-1 \text {. }
$$

Using 2.15 with $\beta=\alpha$, we get

$$
\begin{aligned}
\int_{0}^{h}\left(\int_{y}^{\infty}\left|u_{R}^{\prime}(t)\right|^{\alpha} \mathrm{d} t\right)^{p / \alpha} \mathrm{d} y \lesssim \int_{0}^{h}\left(R^{1-2 \alpha} \chi_{(0,4 R]}(y)\right)^{p / \alpha} \mathrm{d} y \\
\leq R^{(1-2 \alpha) p / \alpha} \min \{h, 4 R\} \text { for all } h, R \in(0, \infty) .
\end{aligned}
$$


Thus, if $\eta \in(0,1]$, then 2.19$)$ and 2.18$)$ imply that

$$
\begin{aligned}
& \int_{0}^{\infty}\left(\int_{0}^{h}\left(\int_{y}^{\infty}\left|u_{R}^{\prime}(\tau)\right|^{\alpha} \mathrm{d} \tau\right)^{p / \alpha} \mathrm{d} y\right) h^{p / \alpha^{\prime}-\eta-1} \mathrm{~d} h \\
& \lesssim R^{(1-2 \alpha) p / \alpha} \int_{0}^{4 R} h^{p / \alpha^{\prime}-\eta} \mathrm{d} h+R^{(1-2 \alpha) p / \alpha+1} \int_{4 R}^{\infty} h^{p / \alpha^{\prime}-\eta-1} \mathrm{~d} h \\
& \quad \approx R^{1-p-\eta} \quad \text { for all } R \in(0, \infty) .
\end{aligned}
$$

Now, we are able to prove (2.14). To this end, we distinguish two cases.

(i) Let $\eta \in(1, p)$. Then, (2.1) with $w(h):=|h|^{-\eta-1},(2.3),(2.17)$ and (2.16) yield

$$
\begin{aligned}
\int_{0}^{\infty} & \int_{0}^{\infty} \frac{\left|u_{R}(x)-u_{R}(y)\right|^{p}}{|x-y|^{\eta+1}} \mathrm{~d} x \mathrm{~d} y \\
& \lesssim \int_{0}^{\infty}\left(\int_{0}^{2 h}\left|u_{R}(y)\right|^{p} \mathrm{~d} y\right) h^{-\eta-1} \mathrm{~d} h+\int_{0}^{\infty}\left(\int_{h}^{\infty}\left|u_{R}^{\prime}(y)\right|^{p} \mathrm{~d} y\right) h^{p-\eta-1} \mathrm{~d} h \\
& \lesssim R^{1-p-\eta} \text { for all } R \in(0, \infty) .
\end{aligned}
$$

(ii) Let $\eta \in(0,1]$. Choose $\alpha \in(1, \infty)$ such that $\alpha^{\prime}>p / \eta$. Then, (2.1) with $w(h):=|h|^{-\eta-1}$, 2.4), (2.20) and (2.16) imply that

$$
\begin{aligned}
\int_{0}^{\infty} \int_{0}^{\infty} \frac{\left|u_{R}(x)-u_{R}(y)\right|^{p}}{|x-y|^{\eta+1}} \mathrm{~d} x \mathrm{~d} y & \\
& \lesssim \int_{0}^{\infty}\left(\int_{0}^{h}\left(\int_{y}^{\infty}\left|u_{R}^{\prime}(\tau)\right|^{\alpha} \mathrm{d} \tau\right)^{p / \alpha} \mathrm{d} y\right) h^{p / \alpha^{\prime}-1-\eta} \mathrm{d} h \\
& +\int_{0}^{\infty}\left(\int_{h}^{\infty}\left|u_{R}^{\prime}(y)\right|^{p} \mathrm{~d} y\right) h^{p-1-\eta} \mathrm{d} h \lesssim R^{1-p-\eta} \quad \text { for all } R \in(0, \infty)
\end{aligned}
$$

Now, we can prove Theorem 1.2 .

Proof of Theorem 1.2. By (2.10), the test function $u_{R}$ satisfies both of conditions (ii), (ii) of Theorem 1.1. We obtain from (1.2), (2.12) and (2.14) that

$$
R^{1-p-\delta} \lesssim C R^{1-p-\eta} \quad \text { for all } R \in(0, \infty)
$$

Since the constant $C$ is independent of $R$, the last estimate implies that $\eta=\delta$.

\section{REFERENCES}

1. P. Gurka and B. Opic, Sharp embeddings of Besov spaces with logarithmic smoothness, Rev. Mat. Complut. 18 (1) (2005), 81-110.

2. P. Gurka and B. Opic, Sharp embeddings of Besov-type spaces, J. Comput. Appl. Math. 208 (2007), 235-269.

3. N. Krugljak, L. Maligranda and L.E. Persson, On an elementary approach to the fractional Hardy inequality, Proc. Amer. Math. Soc. 128 (2000), No. 3, 727-734. 
${ }^{1}$ Department of Mathematics, Czech University of Agriculture, 16521 Prague 6 , Czech Republic.

E-mail address: gurka@tf.czu.cz

2 Mathematical Institute, Academy of Sciences of the Czech Republic, Žitná 25, 11567 Prague 1, Czech Republic;

Department of Mathematics and Didactics of Mathematics, Pedagogical Faculty, Technical University of Liberec, Hálkova 6, 46117 Liberec, Czech RepubLIC.

E-mail address: opic@math.cas.cz 